\title{
The Effect of the Gas-Phase on Differential Inhibition of Intestinal Bacilli
}

\author{
BY J. BRODIE AND W. SHEPHERD \\ Department of Bacteriology, University of St Andrews, Medical \\ School, Dundee
}

SUMMARY : By adjusting the electrolyte content of variously compounded bile-salt media, the growth of coliform bacilli and $R$ forms of intestinal pathogens can be suppressed leaving the $S$ forms of the latter unaffected. Anaerobiosis abolishes the inhibitory effect whereas increased oxygen concentrations enhance it. The degrees of inhibition depend upon the balance of bile salt, electrolyte and oxygen concentration.

Previous experiments (Brodie, 1948) showed that differential inhibitory effects among intestinal bacilli could be obtained when the organisms were grown aerobically in certain media, upon varying the bile salt: electrolyte ratio. The syneretic quality of the gelling agents affected the end result. Similar differential effects with similarly compounded media were obtained in shallow-layer fluid cultures, including fluid gelatin-containing media at $\mathbf{3 7} 7^{\circ}$ (Brodie \& Shepherd, 1949). With the above media, the organisms most resistant to inhibition are the smooth forms of intestinal pathogens. The use of a shallow layer of fluid medium based upon these findings gave an improved isolation rate of intestinal pathogens from morbid material (Brodie, 1949).

It was observed (Brodie \& Shepherd, 1949) that in shake cultures, the higher the concentration of the electrolyte the less tolerant of air the organisms became, with the exception of the smooth form of the Sonne III bacillus; moreover, the lethal effect of bile salt + electrolyte fluid media on the $\mathbf{R}$ and $S$ forms of that organism was influenced more markedly by the depth of the layer of medium rather than by the duration of the exposure to the media. In view of these observations, it was decided to investigate the effects of the gas-phase upon the patterns of differential inhibition.

\section{MATERIALS AND METHODS}

All media were prepared and inoculated as described by Brodie (1948). The following organisms were studied: C1, Bacterium coli communis from human faeces; C2, Bacterium coli communior from human faeces; A1, Aerobacter sp. from milk; A2, Aerobacter sp. from water; SR, a rough form of a recently isolated Sonne III bacillus; SS, a smooth form of the same micro-organism; $R$ and $S$ forms of Flexner bacilli type $V$ (N.C.T.C. 4832), type W (N.C.T.C. 4833), type X (N.C.T.C. 4834), type Y (N.C.T.C. 4836) and type Z (N.C.T.C. 4835); $R$ and $S$ forms of a fresh strain of the Sonne III bacillus. Of these organisms, C1, C2, A1, A2, SR and SS were the strains used previously (Brodie, 1948; Brodie \& Shepherd, 1949).

The gases employed were hydrogen and oxygen, both of commercial standard, 
and specially prepared nitrogen which contained less than 5 p.p.m. of carbon dioxide, no oxygen and no hydrogen (British Oxygen Co.).

Inoculated plates were exposed to the gases in a McIntosh \& Fildes type anaerobic jar (no. 128B, Baskerville \& Lindsay, Manchester). As much air as possible was removed from the jar by a Hyvac oil pump and then filled with the necessary gas; this procedure was repeated six times. When using hydrogen, the last traces of oxygen were burned out in the usual way. In preparing gas mixtures, the jar was filled with the principal gas, the necessary volume was evacuated, as recorded by a mercury manometer, and replaced with the subsidiary gas; a final adjustment of internal pressure was then made before incubation at $87^{\circ}$ for $48 \mathrm{hr}$.

In the experiments with gas mixtures it was found necessary to prepare the mixtures immediately and to make no allowance for a rise in pressure on incubation. Adjustment to atmospheric pressure was made at room temperature immediately after mixing. Only by attention to these details were consistent results obtained.

\section{RESULTS \\ Aerobic experiments}

Initial experiments showed that when the $\mathbf{R}$ and $\mathbf{S}$ forms of the Flexner bacilli were grown aerobically on the surface of $2 \%$ gels made with fibre agar (Thos. Morson and Son, London) containing quarter-strength peptic digest of serum, sodium chloride $(0.5 \% \mathrm{w} / \mathrm{v})$ and taurocholate $(0.5 \% \mathrm{w} / \mathrm{v})$ in the presence of $0.1 \mathrm{M}$ sodium citrate (medium $\mathrm{A}$ ), and $0.2 \mathrm{M}$ sodium citrate (medium B), both $R$ and $S$ forms grew on $A$ but only the $S$ forms on $B$. The $R$ and $S$ forms of the new Sonne III bacillus behaved in the same way. This identity of behaviour by typical representative organisms of the dysentery group suggests that the mechanism of inhibition is common to the group.

The electron-microscope studies already made of strains SR, SS, C1, C2, A1 and A2 (Brodie \& Shepherd, 1949) were extended to the new strains of Flexner and Sonne III bacilli. The $R$ and $S$ forms all behaved in the same way as the $\mathbf{R}$ and $\mathbf{S}$ forms of the original Sonne III bacillus, i.e. the $\mathbf{S}$ forms were unaffected by the higher concentrations of electrolytes in the presence of $0.5 \%(w / v)$ taurocholate whereas the $\mathbf{R}$ forms similarly exposed showed plasmoptysis.

\section{Variation of the gas-phase}

Employing the same agar media as in the aerobic experiments, the effects of different gases, alone and mixed, were examined using the complete range of organisms.

In hydrogen and nitrogen, the coliform bacilli and all the $\mathbf{R}$ and $\mathbf{S}$ forms grew on media $A$ and $B$. In $20 \%(v / v)$ oxygen in nitrogen, the results were the same as obtained under aerobic conditions and of the four coliform bacilli AI and A2 grew on medium A only.

When the oxygen content in nitrogen was increased, the differential properties of both media were enhanced, medium $A$ in $40 \%(v / v)$ oxygen in nitrogen giving results similar to those obtained on medium $B$ in air or $20 \%$ 
$(v / v)$ oxygen in nitrogen. In $60 \%(v / v)$ oxygen in nitrogen medium $B$ was completely inhibitory and medium $A$ behaved as medium $B$ in air or in $20 \%(v / v)$ oxygen in nitrogen. To eliminate the possibility that increased oxygen tension alone was toxic, all the organisms were grown on nutrient agar and nutrient agar with $0.5 \%(\mathrm{w} / \mathrm{v})$ taurocholate under the same experimental conditions and in all instances good growth was obtained.

\section{The effect of the agar gel}

Throughout this work, the $\mathbf{R}$ and $\mathbf{S}$ forms were checked serologically and culturally. During one of these routine checks, a new batch of MacConkey's (1908) bile-salt medium was prepared substituting, as was customary during these investigations, peptically digested sheep's serum for the usual peptone. On this occasion, the agar was changed from fibre agar to Davis agar (Davis Gelatin Co., Christchurch, New Zealand). The alteration in brand of agar gave rise to a dramatic change in colony character of the $\mathbf{R}$ forms. Whereas on the fibre-agar medium these had given typically rough colonies, they now gave, after $24 \mathrm{hr}$. at $37^{\circ}$, colonies which were typically smooth. Picked from Davis agar medium and plated back on the fibre-agar bile-salt medium, the $\mathbf{R}$ forms now grew with their former typically rough colonies. Serial subculture of $\mathbf{R}$ forms on the fibre-agar medium always produced rough colonies; the same procedure on Davis agar medium always produced smooth colonies. Transfer of the $\mathbf{R}$ forms from fibre to Davis agar medium yielded smooth colonies and the reverse transfer yielded rough colonies. This alteration of colony type of the $\mathbf{R}$ forms on the Davis agar medium was not, however, attended by alteration in the serological character of the $R$ forms.

\section{CONCLUSIONS}

From these and previous observations (Brodie, 1948; Brodie \& Shepherd, 1949), it appears that the differential inhibition of $R$ and $S$ forms of dysentery bacilli and certain non-pathogenic intestinal bacilli in fluid and on solid media depends on the following factors.

(a) In fluid media, the depth of the layer, the balance between the bile-salt content and the concentration of the selected electrolyte and the availability of oxygen are the important factors. The consideration governing the selection of the electrolyte is that it must be one of the combinations of an anion and a cation in the ionic series of Hofmeister, the concentration to be used being directly related to its position in the series;

(b) On solid media the above criteria still apply but are contingent on the gel exhibiting a degree of syneresis sufficient to produce the equivalent of a shallow layer of the corresponding fluid medium on the surface of the gel.

The authors wish to thank Prof. W. J. Tulloch for advice and criticism and Prof. G. D. Preston of the Physics Department for permission to use the electronmicroscope on loan from the Department of Scientific and Industrial Research. This paper forms part of the research work undertaken by one of us (J. Brodie) under the terms of the Ernest Hart Memorial Scholarship awarded by the British Medical Association. 


\section{REFERENCES}

Brodie, J. (1948). Observations on the differential inhibition of coliform bacilli and rough variants of intestinal pathogens. J. gen. Microbiol. $2,1$.

Brodie, J. (1949). Shallow-layer fluid enrichment method for intestinal pathogens. J. Path. Bact. 61, 120.

Brodie, J. \& Shepherd, W. (1949). Further observations on the differential inhibition of coliform bacilli and rough variants of intestinal pathogens. J. gen. Microbiol. 3, 74.

MacConkey, A. T. (1908). Bile-salt media and their advantages in some bacteriological examinations. J. Hyg., Camb., 8, 322.

(Received 27 June 1949) 